\title{
ON THE COARSE GEOMETRY OF JAMES SPACES
}

\author{
G. LANCIEN, C. PETITJEAN, AND A. PROCHÁZKA
}

\begin{abstract}
In this note we prove that the Kalton interlaced graphs do not equi-coarsely embed into the James space $\mathcal{J}$ nor into its dual $\mathcal{J}^{*}$. It is a particular case of a more general result on the non equicoarse embeddability of the Kalton graphs into quasi-reflexive spaces with a special asymptotic stucture. This allows us to exhibit a coarse invariant for Banach spaces, namely the non equi-coarse embeddability of this family of graphs, which is very close to but different from the celebrated property $\mathcal{Q}$ of Kalton. We conclude with a remark on the coarse geometry of the James tree space $\mathcal{J} \mathcal{T}$ and of its predual.
\end{abstract}

\section{INTRODUCTION}

In a fundamental paper on the coarse geometry of Banach spaces ([14), N. Kalton introduced a property of metric spaces that he named property $\mathcal{Q}$. In particular, its absence served as an obstruction to coarse embeddability into reflexive Banach spaces. This property is related to the behavior of Lipschitz maps defined on a particular family of metric graphs that we shall denote $\left([\mathbb{N}]^{k}, d_{\mathbb{K}}^{k}\right)_{k \in \mathbb{N}}$. We will recall the precise definitions of these graphs and of property $\mathcal{Q}$ in section 2.2 , Let us just say, vaguely speaking for the moment, that a Banach space $X$ has property $\mathcal{Q}$ if for every Lipschitz map $f$ from $\left([\mathbb{N}]^{k}, d_{\mathbb{K}}^{k}\right)$ to $X$, there exists a full subgraph $[\mathbb{M}]^{k}$ of $[\mathbb{N}]^{k}$, with $\mathbb{M}$ infinite subset of $\mathbb{N}$, on which $f$ satisfies a strong concentration phenomenon. It is then easy to see that if a Banach space $X$ has property $\mathcal{Q}$, then the family of graphs $\left([\mathbb{N}]^{k}, d_{\mathbb{K}}^{k}\right)_{k \in \mathbb{N}}$ does not equi-coarsely embed into $X$ (see the definition in section 2.1). One of the main results in [14 is that any reflexive Banach space has property $\mathcal{Q}$. It then readily follows that a reflexive Banach space cannot contain a coarse copy of all separable metric spaces, or equivalently does not contain a coarse copy of the Banach space $c_{0}$. In fact, with a sophistication of this argument, Kalton proved an even stronger result in 14]: if a separable Banach space contains a coarse copy of $c_{0}$, then there is an integer $k$ such that the dual of order $k$ of $X$ is non separable. In particular, a quasi-reflexive Banach space does not contain a coarse copy of $c_{0}$. However, Kalton proved that the most famous example of a quasireflexive space, namely the James space $\mathcal{J}$, as well as its dual $\mathcal{J}^{*}$, fail property $\mathcal{Q}$.

The main purpose of this paper is to show that, although they do not obey the concentration phenomenon described by property $\mathcal{Q}$, neither $\mathcal{J}$ nor $\mathcal{J}^{*}$ equi-coarsely contains the family of graphs $\left([\mathbb{N}]^{k}, d_{\mathbb{K}}^{k}\right)_{k \in \mathbb{N}}$ (Corollary

2010 Mathematics Subject Classification. 46B20, 46B80, 46B85, 46T99.

The three authors are supported by the French "Investissements d'Avenir" program, project ISITE-BFC (contract ANR-15-IDEX-03). 
5.3). This provides a coarse invariant, namely "not containing equi-coarsely the Kalton graphs", that is very close to but different from property $\mathcal{Q}$. This could allow to find obstructions to coarse embeddability between seemingly close Banach spaces. Our result is actually more general. We prove in Theorem 4.1 that a quasi-reflexive Banach space $X$ such that both $X$ and $X^{*}$ admit an equivalent $p$-asymptotically uniformly smooth norm (see the definition in section 3), for some $p$ in $(1, \infty)$, does not equi-coarsely contain the Kalton graphs.

We conclude this note by showing that if the James tree space $\mathcal{J T}$ or its predual coarsely embeds into a separable Banach space $X$, then there exists $k \in \mathbb{N}$ so that the dual of order $k$ of $X$ is non separable. This extends slightly Theorem 3.5 in [14.

\section{MetriC Notions}

2.1. Coarse embeddings. Let $M, N$ be two metric spaces and $f: M \rightarrow$ $N$ be a map. We define the compression modulus $\rho_{f}$ and the expansion modulus $\omega_{f}$ as follows. For $t \in[0, \infty)$, we set

$$
\begin{aligned}
& \rho_{f}(t)=\inf \left\{d_{N}(f(x), f(y)): d_{M}(x, y) \geq t\right\}, \\
& \omega_{f}(t)=\sup \left\{d_{N}(f(x), f(y)): d_{M}(x, y) \leq t\right\} .
\end{aligned}
$$

We adopt the convention $\sup (\emptyset)=0$ and $\inf (\emptyset)=\infty$. Note that for every $x, y \in M$,

$$
\rho_{f}\left(d_{M}(x, y)\right) \leq d_{N}(f(x), f(y)) \leq \omega_{f}\left(d_{M}(x, y)\right) .
$$

We say that $f$ is a coarse embedding if $\omega_{f}(t)<\infty$ for every $t \in[0,+\infty)$ and $\lim _{t \rightarrow \infty} \rho_{f}(t)=\infty$.

Next, let $\left(M_{i}\right)_{i \in I}$ be a family of metric spaces. We say that the family $\left(M_{i}\right)_{i \in I}$ equi-coarsely embeds into a metric space $N$ if there exist two maps $\rho, \omega:[0,+\infty) \rightarrow[0,+\infty)$ and maps $f_{i}: M_{i} \rightarrow N$ for $i \in I$ such that:

(i) $\lim _{t \rightarrow \infty} \rho(t)=\infty$,

(ii) $\omega(t)<\infty$ for every $t \in[0,+\infty)$,

(iii) $\rho(t) \leq \rho_{f_{i}}(t)$ and $\omega_{f_{i}}(t) \leq \omega(t)$ for every $i \in I$ and $t \in[0, \infty)$.

2.2. The Kalton interlaced graphs and property Q. For $k \in \mathbb{N}$ and $\mathbb{M}$ an infinite subset of $\mathbb{N}$, we put $[\mathbb{M}]^{\leq k}=\{S \subset \mathbb{M}:|S| \leq k\},[\mathbb{M}]^{k}=$ $\{S \subset \mathbb{M}:|S|=k\},[\mathbb{M}]^{\omega}=\{S \subset \mathbb{M}: S$ is infinite $\}$, and $[\mathbb{M}]^{<\omega}=\{S \subset$ $\mathbb{M}: S$ is finite $\}$. We always list the elements of some $\bar{m}$ in $[\mathbb{N}]^{<\omega}$ or in $[\mathbb{N}]^{\omega}$ in increasing order, meaning that if we write $\bar{m}=\left(m_{1}, m_{2}, \ldots, m_{l}\right)$ or $\bar{m}=\left(m_{1}, m_{2}, m_{3}, \ldots\right)$, we tacitly assume that $m_{1}<m_{2}<\cdots$.

For $\bar{m}=\left(m_{1}, m_{2}, \ldots, m_{r}\right) \in[\mathbb{N}]^{<\omega}$ and $\bar{n}=\left(n_{1}, n_{2}, \ldots, n_{s}\right) \in[\mathbb{N}]^{<\omega}$, we write $\bar{m} \prec \bar{n}$, if $r<s \leq k$ and $m_{i}=n_{i}$, for $i=1,2, \ldots, r$, and we write $\bar{m} \preceq \bar{n}$ if $\bar{m} \prec \bar{n}$ or $\bar{m}=\bar{n}$. Thus $\bar{m} \preceq \bar{n}$ if $\bar{m}$ is an initial segment of $\bar{n}$.

Following Kalton [14], for $\mathbb{M} \in[\mathbb{N}]^{\omega}$, we equip $[\mathbb{M}]^{k}$ with a graph structure by declaring $\bar{m} \neq \bar{n} \in[\mathbb{M}]^{k}$ adjacent if and only if

$$
n_{1} \leq m_{1} \leq n_{2} \ldots \leq n_{k} \leq m_{k} \text { or } m_{1} \leq n_{1} \leq m_{2} \ldots \leq m_{k} \leq n_{k} .
$$

For any $\bar{m}, \bar{n} \in[\mathbb{M}]^{k}$, the distance $d_{\mathbb{K}}^{k}(\bar{m}, \bar{n})$ is then defined as the shortest path distance in the graph $[\mathbb{M}]^{k}$. 
Remark 2.1. The distance $d_{\mathbb{K}}^{k}$ is independent of the set $\mathbb{M}$ and therefore $\left[\mathbb{M}_{1}\right]^{k}$ is a metric subspace of $\left[\mathbb{M}_{2}\right]^{k}$ whenever $\mathbb{M}_{1} \in\left[\mathbb{M}_{2}\right]^{\omega}$.

This last claim is an immediate consequence of the following explicit formula for the distance.

Proposition 2.2. Let $k \in \mathbb{N}$ and $\mathbb{M} \in[\mathbb{N}]^{\omega}$. Then $d_{\mathbb{K}}^{k}(\bar{n}, \bar{m})=d(\bar{n}, \bar{m})$ for all $\bar{n}, \bar{m} \in[\mathbb{M}]^{k}$ where $d(\bar{n}, \bar{m})=\sup \{|| \bar{n} \cap S|-| \bar{m} \cap S||: S$ segment of $\mathbb{N}\}$.

Proof. It is easily seen that $d$ is a metric on $[\mathbb{M}]^{k}$. Since $d_{\mathbb{K}}^{k}$ is a graph metric on $[\mathbb{M}]^{k}$, in order to show $d_{\mathbb{K}}^{k}=d$ it is enough to verify that $d_{\mathbb{K}}^{k}(\bar{n}, \bar{m})=1$ if and only if $d(\bar{n}, \bar{m})=1$ and that $d$ is a graph metric.

For $A \subset \mathbb{N}$ let us denote $\mathbf{1}_{A}: \mathbb{N} \rightarrow\{0,1\}$ the indicator function of $A$ and let us first observe the following fact.

Fact: For every $\bar{n}, \bar{m} \in[\mathbb{M}]^{k}$,

$$
d(\bar{n}, \bar{m})=\max _{i} F(i)-\min _{i} F(i)
$$

where $F(i)=F_{\bar{n}, \bar{m}}(i)=\sum_{j=1}^{i} \mathbf{1}_{\bar{n}}(j)-\mathbf{1}_{\bar{m}}(j)$ (and $F(0)=0$ ).

Indeed, we have for any segment $S=[a, b]$ that

$$
|S \cap \bar{n}|-|S \cap \bar{m}|=\sum_{j \in S}\left(\mathbf{1}_{\bar{n}}(j)-\mathbf{1}_{\bar{m}}(j)\right)=F(b)-F(a-1) .
$$

In particular $\max _{S}|| S \cap \bar{n}|-| S \cap \bar{m}|| \leq \max F-\min F$. On the other hand if $S=[a, b]$ is such that $\{F(a-1), F(b)\}=\{\max F, \min F\}$ then ||$S \cap \bar{n}|-| S \cap \bar{m}|| \geq \max F-\min F$ which finishes the proof of the fact.

It is clear that $d_{\mathbb{K}}^{k}(\bar{n}, \bar{m})=1$ if and only if $\max F-\min F=1$. Thus it only remains to prove that $d$ is a graph metric. Now given $\bar{n}, \bar{m}$ such that $d(\bar{n}, \bar{m}) \geq 2$ we are looking for $\bar{\ell} \in[\mathbb{M}]^{k} \backslash\{\bar{m}, \bar{n}\}$ such that $d(\bar{m}, \bar{n})=$ $d(\bar{n}, \bar{\ell})+d(\bar{\ell}, \bar{m})$. Without loss of generality we will assume that $\max F_{\bar{n}, \bar{m}}>$ 0 . Notice that the sets $\arg \max (F)$ and $\arg \min (F)$ are disjoint. We select inductively $\left\{a_{1}<\ldots<a_{p}\right\} \subset \arg \max (F)$ and $\left\{b_{1}<\ldots<b_{q}\right\} \subset \arg \min (F)$ (with $p \geq 1$ and $q \geq 0$ ) with the property that

- $a_{1}=\min \arg \max (F)$,

- For $i \geq 1, b_{i}=\min \left(\left\{n>a_{i}\right\} \cap \arg \min (F)\right)$, if this is not empty.

- $a_{i+1}=\min \left(\left\{n>b_{i}\right\} \cap \arg \max (F)\right)$, if this set is not empty.

Notice that $\left\{a_{1}, \ldots, a_{p}\right\} \subset \bar{n} \backslash \bar{m}$ and $\left\{b_{1}, \ldots, b_{q}\right\} \subset \bar{m} \backslash \bar{n}$. Notice also that either $p=q$ or $p=q+1$. In the latter case we define $b_{p}:=r$ for some $r$ such that $r>a_{p}$ and $F(r-1)>F(r)$. Such $r$ must exist since $F\left(\max \left\{n_{k}, m_{k}\right\}\right)=0$. Also we have $r \in \bar{m} \backslash \bar{n}$. We will set

$$
\bar{\ell}=\bar{n} \cup\left\{b_{1}, \ldots, b_{p}\right\} \backslash\left\{a_{1}, \ldots, a_{p}\right\} .
$$

It is clear that $\bar{\ell} \in[\mathbb{M}]^{k}$. We also have $\max F_{\bar{\ell}, \bar{m}}=\max F_{\bar{n}, \bar{m}}-1$ and $\min F_{\bar{\ell}, \bar{m}}=\min F_{\bar{n}, \bar{m}}$. Indeed, the point $\bar{\ell}$ is constructed in such a way that when $F_{\bar{n}, \bar{m}}$ attains its maximum for the first time (going from the left), $F_{\bar{\ell}, \bar{m}}$ is reduced by one and stays reduced by 1 until the next time the minimum of $F_{\bar{n}, \bar{m}}$ is attained (or until the point $r$ ) where this reduction is corrected back; and so on. Thus $d(\bar{\ell}, \bar{m})=d(\bar{n}, \bar{m})-1$. Also, since the sets $\left\{a_{1}, \ldots, a_{p}\right\}$ and $\left\{b_{1}, \ldots, b_{p}\right\}$ are interlaced we have $F_{\bar{n}, \bar{m}}-1 \leq F_{\bar{\ell}, \bar{m}} \leq F_{\bar{n}, \bar{m}}$. Therefore, since 
$F_{\bar{n}, \bar{m}}=F_{\bar{n}, \bar{\ell}}+F_{\bar{\ell}, \bar{m}}$, we have that $0 \leq F_{\bar{n}, \bar{\ell}} \leq 1$ and so finally $d(\bar{n}, \bar{\ell})=1$, since it is clear that $\bar{n} \neq \bar{\ell}$.

Note that if $X$ is a Banach space and $f:\left([\mathbb{M}]^{k}, d_{\mathbb{K}}^{k}\right) \rightarrow X$ is a map with finite expansion modulus $\omega_{f}$, then $\omega_{f}(1)$ is actually the Lipschitz constant of $f$ as $d_{\mathbb{K}}^{k}$ is a graph distance on $[\mathbb{M}]^{k}$.

In [14] the property $\mathcal{Q}$ is defined in the setting of metric spaces. For homogeneity reasons, its definition can be simplified for Banach spaces. Let us recall it here.

Definition 2.3. Let $X$ be a Banach space. We say that $X$ has property $\mathcal{Q}$ if there exists $C \geq 1$ such that for every $k \in \mathbb{N}$ and every Lipschitz map $f:\left([\mathbb{N}]^{k}, d_{\mathbb{K}}^{k}\right) \rightarrow X$, there exists an infinite subset $\mathbb{M}$ of $\mathbb{N}$ such that:

$$
\forall \bar{n}, \bar{m} \in[\mathbb{M}]^{k},\|f(\bar{n})-f(\bar{m})\| \leq C \omega_{f}(1) .
$$

The following proposition should be clear from the definitions. We shall however include its short proof.

Proposition 2.4. Let $X$ be a Banach space. If $X$ has property $\mathcal{Q}$, then the family of graphs $\left([\mathbb{N}]^{k}, d_{\mathbb{K}}^{k}\right)_{k \in \mathbb{N}}$ does not equi-coarsely embed into $X$.

Proof. Let $C \geq 1$ be given by the definition of property $\mathcal{Q}$. Aiming for a contradiction, assume that the family $\left([\mathbb{N}]^{k}, d_{\mathbb{K}}^{k}\right)_{k \in \mathbb{N}}$ equi-coarsely embeds into $X$. That is, there are maps $f_{k}:\left([\mathbb{N}]^{k}, d_{\mathbb{K}}^{k}\right) \rightarrow X$ and two functions $\rho, \omega:[0,+\infty) \rightarrow[0,+\infty)$ such that $\lim _{t \rightarrow \infty} \rho(t)=\infty$ and

$$
\forall k \in \mathbb{N} \quad \forall t>0 \quad \rho(t) \leq \rho_{f_{k}}(t) \text { and } \omega_{f_{k}}(t) \leq \omega(t)<\infty .
$$

Thus, for every $k \in \mathbb{N}$, there exists an infinite subset $\mathbb{M}_{k}$ of $\mathbb{N}$ such that $\left.\operatorname{diam}\left(f\left(\left[\mathbb{M}_{k}\right]^{k}\right)\right)\right) \leq C \omega(1)$. Since diam $\left(\left[\mathbb{M}_{k}\right]^{k}\right)=k$, this implies that for all $k \in \mathbb{N}, \rho(k) \leq C \omega(1)$. This contradicts the fact that $\lim _{t \rightarrow \infty} \rho(t)=\infty$.

A concrete bi-Lipschitz copy of the metric spaces $\left([\mathbb{N}]^{k}, d_{\mathbb{K}}^{k}\right)$ in $c_{0}$ is given by the following proposition.

Proposition 2.5. Let $\left(s_{n}\right)_{n=1}^{\infty}$ be the summing basis of $c_{0}$, that is $s_{n}=\sum_{i=1}^{n} e_{i}$, where $\left(e_{i}\right)_{i=1}^{\infty}$ is the canonical basis of $c_{0}$.

For $k \in \mathbb{N}$, define $f_{k}:\left([\mathbb{N}]^{k}, d_{\mathbb{K}}^{k}\right) \rightarrow c_{0}$ by $f_{k}(\bar{n})=\sum_{i=1}^{k} s_{n_{i}}$. Then

$$
\frac{1}{2} d_{\mathbb{K}}^{k}(\bar{n}, \bar{m}) \leq\left\|f_{k}(\bar{n})-f_{k}(\bar{m})\right\|_{\infty} \leq d_{\mathbb{K}}^{k}(\bar{n}, \bar{m})
$$

for all $\bar{n}, \bar{m} \in[\mathbb{N}]^{k}$.

Proof. Since $d_{\mathbb{K}}^{k}=d$, one can show (as in the Fact in the proof of Proposition 2.2) that $d_{\mathbb{K}}^{k}(\bar{n}, \bar{m})=\max \left(f_{k}(\bar{n})-f_{k}(\bar{m})\right)-\min \left(f_{k}(\bar{n})-f_{k}(\bar{m})\right)$. The result then follows easily since $\min \left(f_{k}(\bar{n})-f_{k}(\bar{m})\right) \leq 0 \leq \max \left(f_{k}(\bar{n})-f_{k}(\bar{m})\right)$ for all $\bar{n}, \bar{m} \in[\mathbb{N}]^{k}$.

Remark 2.6. We already explained that $c_{0}$ cannot coarsely embed into any Banach space with property $\mathcal{Q}$ (in particular into any reflexive Banach space) and that Kalton even showed with additional arguments that if $c_{0}$ coarsely embeds into a separable Banach space $X$, then one of the iterated 
duals of $X$ has to be non separable. An inspection of his proof shows that the uniformly discrete metric spaces

$$
M_{k}=\left\{\sum_{i=1}^{k} s_{n_{i}} \times \mathbf{1}_{A}:\left(n_{1}, \ldots, n_{k}\right) \in[\mathbb{N}]^{k}, A \in[\mathbb{N}]^{\omega}\right\} \subset c_{0}
$$

do not equi-coarsely embed into any Banach space $X$ such that $X^{(r)}$ is separable for all $r$. See Theorem 6.1 below for more on this subject.

Studying further the property $\mathcal{Q}$ in [14], Kalton exhibited non reflexive quasi-reflexive spaces with the property $\mathcal{Q}$ but showed that $\mathcal{J}$ and $\mathcal{J}^{*}$ fail property $\mathcal{Q}$. It is worth noticing that a theorem of Schoenberg [21] implies that $\ell_{1}$ coarsely embeds into $\ell_{2}$, and therefore $\ell_{1}$ provides a simple example of a non-reflexive Banach space with property $\mathcal{Q}$.

We conclude this section with two propositions that we state here for future reference. We start with a classical version of Ramsey's theorem.

Proposition 2.7 (Corollary 1.2 in [10]). Let $(K, d)$ be a compact metric space, $k \in \mathbb{N}$ and $f:[\mathbb{N}]^{k} \rightarrow K$. Then for every $\varepsilon>0$, there exists an infinite subset $\mathbb{M}$ of $\mathbb{N}$ such that $d(f(\bar{n}), f(\bar{m}))<\varepsilon$ for every $\bar{n}, \bar{m} \in[\mathbb{M}]^{k}$.

For a Banach space $X$, we call tree of height $k$ in $X$ any family $(x(\bar{n}))_{\bar{n} \in[\mathbb{N}] \leq k}$, with $x(\bar{n}) \in X$. Then, if $\mathbb{M} \in[\mathbb{N}]^{\omega},(x(\bar{n}))_{\bar{n} \in[\mathbb{M}] \leq k}$ will be called a full subtree of $(x(\bar{n}))_{\bar{n} \in[\mathbb{N}] \leq k}$. A tree $\left(x^{*}(\bar{n})\right)_{\bar{n} \in[\mathbb{M}] \leq k}$ in $X^{*}$ is called weak $k^{*}$-null if for any $\bar{n} \in[\mathbb{M}] \leq k-1$, the sequence $\left(x^{*}\left(n_{1}, \ldots, n_{k-1}, t\right)\right)_{t>n_{k-1}, t \in \mathbb{M}}$ is weak ${ }^{*}$-null.

The next proposition is based on a weak*-compactness argument and will be crucial for our proofs. Although the distance considered on $[\mathbb{N}]^{k}$ is different, the proof follows the same lines as Lemma 4.1 in [3]. We therefore state it now without further detail.

Proposition 2.8. Let $X$ be a separable Banach space, $k \in \mathbb{N}$, and $f$ : $\left([\mathbb{N}]^{k}, d_{\mathbb{K}}^{k}\right) \rightarrow X^{*}$ a Lipschitz map. Then there exist $\mathbb{M} \in[\mathbb{N}]^{\omega}$ and a weak ${ }^{*}$ null tree $\left(x^{*}(\bar{m})\right)_{\bar{m} \in[\mathbb{M}] \leq k}$ in $X^{*}$ with $\left\|x_{\bar{m}}^{*}\right\| \leq \omega_{f}(1)$ for all $\bar{m} \in[\mathbb{M}] \leq k \backslash\{\emptyset\}$ and so that

$$
\forall \bar{n} \in[\mathbb{M}]^{k}, f(\bar{n})=\sum_{i=0}^{k} x^{*}\left(n_{1}, \ldots, n_{i}\right)=\sum_{\bar{m} \preceq \bar{n}} x^{*}(\bar{m}) .
$$

\section{UNIFORM ASYMPTOTIC PROPERTIES OF NORMS AND RELATED ESTIMATES}

We recall the definitions that will be considered in this paper. For a Banach space $(X,\|\|)$ we denote by $B_{X}$ the closed unit ball of $X$ and by $S_{X}$ its unit sphere. The following definitions are due to V. Milman [19] and we adopt the notation from [13]. For $t \in[0, \infty)$ we define

$$
\bar{\rho}_{X}(t)=\sup _{x \in S_{X}} \inf _{Y} \sup _{y \in S_{Y}}(\|x+t y\|-1),
$$

where $Y$ runs through all closed subspaces of $X$ of finite codimension. Then, the norm \|\| is said to be asymptotically uniformly smooth (in short AUS) if

$$
\lim _{t \rightarrow 0} \frac{\bar{\rho}_{X}(t)}{t}=0
$$


For $p \in(1, \infty)$ it is said to be p-asymptotically uniformly smooth (in short $p$-AUS) if there exists $c>0$ such that for all $t \in[0, \infty), \bar{\rho}_{X}(t) \leq c t^{p}$.

We will also need the dual modulus defined by

$$
\bar{\delta}_{X}^{*}(t)=\inf _{x^{*} \in S_{X^{*}}} \sup _{E} \inf _{y^{*} \in S_{E}}\left(\left\|x^{*}+t y^{*}\right\|-1\right),
$$

where $E$ runs through all finite-codimensional weak*-closed subspaces of $X^{*}$. The norm of $X^{*}$ is said to be weak asymptotically uniformly convex (in short $\left.\mathrm{AUC}^{*}\right)$ if $\bar{\delta}_{X}^{*}(t)>0$ for all $t$ in $(0, \infty)$. If there exists $c>0$ and $q \in[1, \infty)$ such that for all $t \in[0,1] \bar{\delta}_{X}^{*}(t) \geq c t^{q}$, we say that the norm of $X^{*}$ is $q$-AUC*. The following proposition is elementary.

Proposition 3.1. Let $X$ be a Banach space. For any $t \in(0,1)$, any weakly null sequence $\left(x_{n}\right)_{n=1}^{\infty}$ in $B_{X}$ and any $x \in S_{X}$ we have:

$$
\limsup _{n \rightarrow \infty}\left\|x+t x_{n}\right\| \leq 1+\bar{\rho}_{X}(t) .
$$

For any weak $k^{*}$-null sequence $\left(x_{n}^{*}\right)_{n=1}^{\infty} \subset X^{*}$ and for any $x^{*} \in X^{*} \backslash\{0\}$ we have

$$
\limsup _{n \rightarrow \infty}\left\|x^{*}+x_{n}^{*}\right\| \geq\left\|x^{*}\right\|\left(1+\bar{\delta}_{X}^{*}\left(\frac{\limsup \left\|x_{n}^{*}\right\|}{\left\|x^{*}\right\|}\right)\right) .
$$

We will also need the following refinement (see Proposition 2.1 in [18]).

Proposition 3.2. Let $X$ be a Banach space. Then the bidual norm on $X^{* *}$ has the following property. For any $t \in(0,1)$, any weak $k^{*}$-null sequence $\left(x_{n}^{* *}\right)_{n=1}^{\infty}$ in $B_{X^{* *}}$ and any $x \in S_{X}$ we have:

$$
\limsup _{n \rightarrow \infty}\left\|x+t x_{n}^{* *}\right\| \leq 1+\bar{\rho}_{X}(t)
$$

Let us now recall the following classical duality result concerning these moduli (see for instance [8] Corollary 2.3 for a precise statement).

Proposition 3.3. Let $X$ be a Banach space. Then \|\|$_{X}$ is AUS if and and only if \|\|$_{X^{*}}$ is $A U C^{*}$.

If $p, q \in(1, \infty)$ are conjugate exponents, then \|\|$_{X}$ is $p$-AUS if and and only if \|\|$_{X^{*}}$ is $q-A U C^{*}$.

We conclude this section with a list of a few classical properties of Orlicz functions and norms that are related to these moduli. A map $\varphi:[0, \infty) \rightarrow$ $[0, \infty)$ is called an Orlicz function if it is continuous, non decreasing, convex and so that $\varphi(0)=0$ and $\lim _{t \rightarrow \infty} \varphi(t)=\infty$. The Orlicz norm \|\|$_{\ell_{\varphi}}$, associated with $\varphi$ is defined on $c_{00}$, the space of finitely supported sequences, as follows:

$$
\forall x=\left(x_{n}\right)_{n=1}^{\infty} \in c_{00}, \quad\|x\|_{\ell \varphi}=\inf \left\{r>0, \sum_{n=1}^{\infty} \varphi\left(x_{n} / r\right) \leq 1\right\} .
$$

The following is immediate from the definition.

Lemma 3.4. Let $\varphi:[0, \infty) \rightarrow[0, \infty)$ be an Orlicz function and $p \in[1, \infty)$.

(i) If there exists $C>0$ such that $\varphi(t) \leq C t^{p}$, for all $t \in[0,1]$, then there exists $A>0$ such that $\|x\|_{\ell_{\varphi}} \leq A\|x\|_{\ell_{p}}$, for all $x \in c_{00}$.

(ii) If there exists $c>0$ such that $\varphi(t) \geq c t^{p}$, for all $t \in[0,1]$, then there exists $a>0$ such that $\|x\|_{\ell_{\varphi}} \geq a\|x\|_{\ell_{p}}$, for all $x \in c_{00}$. 
Assume now that $\varphi:[0, \infty) \rightarrow[0, \infty)$ is an Orlicz function which is 1 Lipschitz and such that $\lim _{t \rightarrow \infty} \varphi(t) / t=1$. Consider for $(s, t) \in \mathbb{R}^{2}$,

$$
N_{2}^{\varphi}(s, t)= \begin{cases}|s|+|s| \varphi(|t| /|s|) & \text { if } s \neq 0, \\ |t| & \text { if } s=0 .\end{cases}
$$

Then define by induction for all $n \geq 3$ :

$$
\forall\left(s_{1}, \ldots, s_{n}\right) \in \mathbb{R}^{n}, N_{n}^{\varphi}\left(s_{1}, \ldots, s_{n}\right)=N_{2}^{\varphi}\left(N_{n-1}^{\varphi}\left(s_{1}, \ldots, s_{n-1}\right), s_{n}\right) .
$$

The following is proved in [16] (see Lemma 4.3 and its preparation).

\section{Lemma 3.5.}

(i) For any $n \geq 2$, the function $N_{n}^{\varphi}$ is an absolute (or lattice) norm on $\mathbb{R}^{n}$, meaning that $N_{n}\left(s_{1}, \ldots, s_{n}\right) \leq N_{n}\left(t_{1}, \ldots, t_{n}\right)$, whenever $\left|s_{i}\right| \leq\left|t_{i}\right|$ for all $i \leq n$.

(ii) For any $n \in \mathbb{N}$ and any $x \in \mathbb{R}^{n}$ :

$$
\frac{1}{2}\|s\|_{\ell_{\varphi}} \leq N_{n}^{\varphi}(s) \leq e\|s\|_{\ell_{\varphi}} .
$$

When $X$ is a Banach space, it is easy to see that $\bar{\rho}_{X}$ is a 1-Lipschitz Orlicz function such that $\lim _{t \rightarrow \infty} \rho(t) / t=1$. But due to its lack of convexity, $\bar{\delta}_{X}^{*}$ is not an Orlicz function and we need to modify it. Following [16, we define

$$
\delta(t)=\int_{0}^{t} \frac{\bar{\delta}_{X}^{*}(s)}{s} d s .
$$

It is easy to see that $\bar{\delta}_{X}^{*}(t) / t$ is increasing and tends to 1 as $t$ tends to $\infty$. Therefore, $\delta$ is an Orlicz function which is 1-Lipschitz, such that $\lim _{t \rightarrow \infty} \delta(t) / t=$ 1 and satisfying:

$$
\forall t \in[0, \infty), \quad \bar{\delta}_{X}^{*}(t / 2) \leq \delta(t) \leq \bar{\delta}_{X}^{*}(t) .
$$

The following statement is now a direct consequence of Lemmas 3.4 and 3.5 .

Lemma 3.6. Let $X$ be a Banach space and $p \in[1, \infty)$.

(i) If there exists $C>0$ such that $\bar{\rho}_{X}(x) \leq C t^{p}$, for all $t \in[0,1]$, then there exists $A>0$ such that

$$
\forall n \in \mathbb{N} \forall x \in \mathbb{R}^{n}, \quad N_{n}^{\bar{\rho}_{X}}(x) \leq A\|x\|_{\ell_{p}^{n}} .
$$

(ii) If there exists $c>0$ such that $\bar{\delta}_{X}^{*}(t) \geq c t^{p}$, for all $t \in[0,1]$, then there exists $a>0$ such that

$$
\forall n \in \mathbb{N} \forall x \in \mathbb{R}^{n}, \quad N_{n}^{\delta}(x) \geq a\|x\|_{\ell_{p}^{n}} .
$$

We will also use the following reformulation of Propositions 3.1 and 3.2 in terms of the norms $N_{2}^{\delta}$ and $N_{2}^{\bar{\rho}_{X}}$.

Lemma 3.7. Let $X$ be a Banach space.

(i) Let $\left(x_{n}^{*}\right) \subset X^{*}$ be weak $k^{*}$-null. Then for any $x^{*} \in X^{*}$ we have

$$
\limsup _{n \rightarrow \infty}\left\|x^{*}+x_{n}^{*}\right\| \geq N_{2}^{\delta}\left(\left\|x^{*}\right\|, \lim \sup \left\|x_{n}^{*}\right\|\right) .
$$

(ii) Similarly, if $\left(x_{n}^{* *}\right) \subset X^{* *}$ is weak ${ }^{*}$-null and $x \in X$, then

$$
\liminf _{n \rightarrow \infty}\left\|x+x_{n}^{* *}\right\| \leq N_{2}^{\bar{\rho}_{X}}\left(\|x\|, \liminf \left\|x_{n}^{* *}\right\|\right) .
$$


Proof. If $x^{*}=0$ there is nothing to do, so we may assume that $x^{*} \neq 0$. By application of Proposition 3.1 we see that

$$
\begin{aligned}
\limsup _{n \rightarrow \infty}\left\|x^{*}+x_{n}^{*}\right\| & \geq\left\|x^{*}\right\|\left(1+\bar{\delta}_{X}^{*}\left(\frac{\limsup \left\|x_{n}^{*}\right\|}{\left\|x^{*}\right\|}\right)\right) \\
& \geq\left\|x^{*}\right\|\left(1+\delta\left(\frac{\lim \sup _{\|}\left\|x_{n}^{*}\right\|}{\left\|x^{*}\right\|}\right)\right)=N_{2}^{\delta}\left(\left\|x^{*}\right\|, \lim \sup \left\|x_{n}^{*}\right\|\right)
\end{aligned}
$$

The proof of the second claim is even simpler so we leave it to the reader.

\section{The General Result}

Let us first recall that a Banach space is said to be quasi-reflexive if the image of its canonical embedding into its bidual is of finite codimension in its bidual. We can now state our main result.

Theorem 4.1. Let $X$ be a quasi-reflexive Banach space, let $p \in(1, \infty)$ and denote $q$ its conjugate exponent. Assume that $X$ admits an equivalent $p$ AUS norm and that $X^{*}$ admits an equivalent $q$-AUS norm. Then the family $\left([\mathbb{N}]^{k}, d_{\mathbb{K}}^{k}\right)_{k \in \mathbb{N}}$ does not equi-coarsely embed into $X^{* *}$.

We immediately deduce the following.

Corollary 4.2. Let $X$ be a quasi-reflexive Banach space, let $p \in(1, \infty)$ and denote $q$ its conjugate exponent. Assume that $X$ admits an equivalent $p$ AUS norm and that $X^{*}$ admits an equivalent $q-A U S$ norm. Then the family $\left([\mathbb{N}]^{k}, d_{\mathbb{K}}^{k}\right)_{k \in \mathbb{N}}$ does not equi-coarsely embed into $X$, nor does it equi-coarsely embed into any iterated dual $X^{(r)}(r \geq 0)$ of $X$.

Proof. Since $X$ is quasi reflexive we infer that $X^{(r)}$ admits an equivalent $p$-AUS norm when $r$ is even and it admits an equivalent $q$-AUS norm when $r$ is odd. Indeed, note that when $r$ is even $X^{(r)}$ is isomorphic to $X \oplus_{p} F$ where $F$ is finite-dimensional (resp. $X^{(r)} \simeq X^{*} \oplus_{q} F$ when $r$ is odd). Now it is obvious from Theorem 4.1 that $\left([\mathbb{N}]^{k}\right)_{k \in \mathbb{N}}$ do not equi-coarsely embed into $X^{(r)}$ when $r$ is even. When $r$ is odd, we just exchange the roles of $p$ and $q$.

Before going into the detailed proof of Theorem 4.1 let us briefly indicate the main idea. We assume that there is an equi-coarse family of embeddings $\left(f_{k}\right)$ of $[\mathbb{N}]^{k}$ into $X^{* *}$ with moduli $\rho$ and $\omega$. We fix $k$ sufficiently large and observe that, up to passing to a subgraph, $f_{k}$ can be represented as the sum along the branches of a weak*-null countably branching tree of height $k$, say $\left(z_{\bar{n}}\right)_{\bar{n} \in[N] \leq k}$. Moreover the norms of the elements of this tree stabilize on each level towards values $\left(K_{i}\right)_{i=1}^{k} \subset[0, \omega(1)]$. Applying the existence of a $q-A U S$ norm on $X^{*}$ one can show that $\sum_{i=1}^{k} K_{i}^{p} \leq c^{p} \omega(1)^{p}$ where $c$ is a constant depending only on $X$. The benefit of this observation is twofold. On one hand we will be able to construct two elements $\bar{n}_{0}, \bar{m}_{0} \in[\mathbb{N}]^{l}$ (with $l \leq k$ ) such that $\sum_{i=1}^{l} z_{\left(n_{1}, \ldots, n_{i}\right)}-z_{\left(m_{1}, \ldots, m_{i}\right)}$ is small in norm (say less than $2 c \omega(1)$ ) while $d_{\mathbb{K}}^{l}\left(\bar{n}_{0}, \bar{m}_{0}\right)$ is large (say $\left.\rho\left(d_{\mathbb{K}}^{l}\left(n_{0}, \bar{m}_{0}\right)\right)>3 c \omega(1)\right)$. On the other hand the $p-A U S$ renormability of $X$ together with the quasi-reflexivity allows 
to extend these elements to elements $\bar{n}, \bar{m} \in[\mathbb{N}]^{k}$ such that $d_{\mathbb{K}}^{k}(\bar{n}, \bar{m})$ is still large and

$$
\begin{aligned}
\left\|\sum_{i=l+1}^{k} z_{\left(n_{1}, \ldots, n_{i}\right)}-z_{\left(m_{1}, \ldots, m_{i}\right)}\right\| & \sim\left(\sum_{i=l+1}^{k}\left\|z_{\left(n_{1}, \ldots, n_{i}\right)}-z_{\left(m_{1}, \ldots, m_{i}\right)}\right\|^{p}\right)^{1 / p} \\
& \sim\left(\sum_{i=l+1}^{k} K_{i}^{p}\right)^{1 / p} \leq c \omega(1) .
\end{aligned}
$$

Eventually, summing the tree from 1 to $k$ over the branches ending by $\bar{n}$ and $\bar{m}$ we get the desired contradiction

$$
3 c \omega(1)<\rho\left(d_{\mathbb{K}}^{k}(\bar{n}, \bar{m})\right) \leq\left\|f_{k}(\bar{n})-f_{k}(\bar{m})\right\| \leq 3 c \omega(1) .
$$

Proof of Theorem 4.1. Let us assume that there are two maps $\rho, \omega:[0,+\infty) \rightarrow$ $[0,+\infty)$ and maps $f_{k}\left([\mathbb{N}]^{k}, d_{\mathbb{K}}^{k}\right): \rightarrow\left(X^{* *},\|\|\right)$ for $k \in \mathbb{N}$ such that:

(i) $\lim _{t \rightarrow \infty} \rho(t)=\infty$,

(ii) $\omega(t)<\infty$ for every $t \in(0,+\infty)$,

(iii) $\rho(t) \leq \rho_{f_{k}}(t)$ and $\omega_{f_{k}}(t) \leq \omega(t)$ for every $k \in \mathbb{N}$ and $t \in(0, \infty)$.

Note that all $f_{k}$ 's are $\omega(1)$-Lipschitz for \|\| and so $\omega(1)>0$. Since all the sets $[\mathbb{N}]^{k}$ are countable, we may and will assume that $X$ and therefore, by the quasi-reflexivity of $X$, that all its iterated duals are separable.

Let us fix $N \in \mathbb{N}$. Pick $\alpha \in \mathbb{N}$ such that $\alpha \geq \frac{p}{q}$ and set $k=N^{1+\alpha} \in \mathbb{N}$. We also fix $\eta>0$. We shall provide at the end of our proof a contradiction if $N$ is chosen large enough and $\eta$ small enough. We denote \|\| the original norm on $X$, as well as its dual and bidual norms. Let us assume, as we may, that \|\| is $p$-AUS on $X$. We denote its modulus of asymptotic uniform smoothness $\bar{\rho}_{\|\|}$or simply $\bar{\rho}_{X}$.

For the first step of the proof we shall exploit the existence of an equivalent $q$-AUS norm || on $X^{*}$ (we also denote || its dual norm on $X^{* *}$ ). It is worth mentioning that if $X$ is not reflexive, || cannot be the dual norm of an equivalent norm on $X$ (see for instance Proposition 2.6 in [7]). Assume also that there exists $b>0$ such that

$$
\forall z \in X^{* *} \quad b\|z\| \leq|z| \leq\|z\| .
$$

Then we have that all $f_{k}$ 's are also $\omega(1)$-Lipschitz for || .

By Proposition 3.3, we have that there exists $c>0$ such that for all $t \in[0,1]$, $\bar{\delta}_{||}^{*}(t) \geq c t^{p}$. We denote again

$$
\delta(t)=\int_{0}^{t} \frac{\bar{\delta}_{||}^{*}(s)}{s} d s .
$$

Recall that Lemma 3.6 ensures the existence of $a>0$ such that for all $n \in \mathbb{N}$, $N_{n}^{\delta} \geq 2 a\|\|_{\ell_{p}^{n}}$.

First, using the separability of $X^{*}$ and Proposition 2.8, we may assume by passing to a full subtree, that there exist a weak ${ }^{*}$-null tree $(z(\bar{m}))_{\bar{m} \in[\mathbb{N}] \leq k}$ in $X^{* *}$ with $\left|z_{\bar{m}}\right| \leq \omega(1)$ for all $\bar{m} \in[\mathbb{N}] \leq k \backslash\{\emptyset\}$ and so that

$$
\forall \bar{n} \in[\mathbb{N}]^{k}, f_{k}(\bar{n})=\sum_{i=0}^{k} z\left(n_{1}, \ldots, n_{i}\right)=\sum_{\bar{m} \preceq \bar{n}} z(\bar{m}) .
$$


For $r \in \mathbb{N}$ we denote $E_{r}=\left\{\bar{m}=\left(m_{1}, \ldots, m_{j}\right) \in[\mathbb{N}] \leq k \backslash\{\emptyset\}, m_{j}=r\right\}$ and $F_{r}=\bigcup_{u=1}^{r} E_{u}$. Fix a sequence $\left(\lambda_{r}\right)_{r=1}^{\infty}$ in $(0,1)$ such that $\prod_{r=1}^{\infty} \lambda_{r}>\frac{1}{2}$. We now use Lemma 3.7 (i) and the fact that $(z(\bar{m}))_{\bar{m} \in[\mathbb{N}] \leq k}$ is a weak*-null tree to build inductively $n_{1}<\ldots<n_{r}$ so that for all $\bar{n}^{1}, \ldots, \bar{n}^{L} \in F_{n_{r}-1}$, for all $\varepsilon_{1}, \ldots, \varepsilon_{L} \in\{-1,1\}$ and all $\bar{n} \in E_{n_{r}}$, we have

$$
\left|z(\bar{n})+\sum_{l=1}^{L} \varepsilon_{l} z\left(\bar{n}^{l}\right)\right| \geq \lambda_{r} N_{2}^{\delta}\left(\left|\sum_{l=1}^{L} \varepsilon_{l} z\left(\bar{n}^{l}\right)\right|,|z(\bar{n})|\right) .
$$

Therefore, using the fact that $N_{2}^{\delta}$ is an absolute norm and after passing to a full subtree, we may assume that for all $r_{1}<\cdots<r_{L}$ in $\mathbb{N}$, all $\varepsilon_{1}, \ldots, \varepsilon_{L} \in$ $\{-1,1\}$ and all $\bar{n}^{1}, \ldots, \bar{n}^{L}$ so that $\bar{n}^{l} \in E_{r_{l}}$ for $1 \leq l \leq L$, we have

$$
\left|\sum_{l=1}^{L} \varepsilon_{l} z\left(\bar{n}^{l}\right)\right| \geq \frac{1}{2} N_{L}^{\delta}\left(\left|z\left(\bar{n}^{1}\right)\right|, \ldots,\left|z\left(\bar{n}^{L}\right)\right|\right) \geq a\left(\sum_{i=1}^{L}\left|z\left(\bar{n}^{l}\right)\right|^{p}\right)^{1 / p} .
$$

Assume now that $\bar{n}=\left(n_{1}, \ldots, n_{k}\right) \in \mathbb{N}^{k}$ is such that $n_{1}<\cdots<n_{k}$ are even and choose $\bar{m}=\left(m_{1}, \ldots, m_{k}\right)$ so that $n_{1}<m_{1}<\cdots<n_{k}<m_{k}$. It follows from (4.2) that

$$
\begin{aligned}
|f(\bar{n})-f(\bar{m})|= & \left|\sum_{i=1}^{k} z\left(n_{1}, \ldots, n_{i}\right)-z\left(m_{1}, \ldots, m_{i}\right)\right| \\
& \geq a\left(\sum_{i=1}^{k}\left|z\left(n_{1}, \ldots, n_{i}\right)\right|^{p}+\left|z\left(m_{1}, \ldots, m_{i}\right)\right|^{p}\right)^{1 / p} .
\end{aligned}
$$

We now use the fact that $d_{\mathbb{K}}^{k}(\bar{n}, \bar{m})=1$ and $f$ is $\omega(1)$-Lipschitz, to deduce

$$
\left(\sum_{i=1}^{k}\left|z\left(n_{1}, \ldots, n_{i}\right)\right|^{p}\right)^{1 / p} \leq \frac{1}{a} \omega(1) .
$$

So replacing $\mathbb{N}$ with $2 \mathbb{N}$ and setting $A=1 / a$, we may assume that

$$
\forall \bar{n} \in[\mathbb{N}]^{k}, \quad\left(\sum_{i=1}^{k}\left|z\left(n_{1}, \ldots, n_{i}\right)\right|^{p}\right)^{1 / p} \leq A \omega(1) .
$$

By Ramsey's theorem (Proposition 2.7), we may also assume, after passing again to a full subtree, that for all $i \in\{1, \ldots, k\}$ there exists $K_{i} \in$ $[0, \omega(1)]$ such that

$$
\forall\left(n_{1}, \ldots, n_{i}\right) \in[\mathbb{N}]^{i}, K_{i} \leq\left|z\left(n_{1}, \ldots, n_{i}\right)\right| \leq K_{i}+\eta .
$$

The estimate (4.3) yields

$$
\sum_{i=1}^{k} K_{i}^{p} \leq A^{p} \omega(1)^{p}
$$

Therefore, since $k=N^{1+\alpha}$, there exists $j \in\left\{0, N, \ldots, N\left(N^{\alpha}-1\right)\right\}$ such that

$$
\sum_{i=j+1}^{j+N} K_{i}^{p} \leq \frac{A^{p} \omega(1)^{p}}{N^{\alpha}} \text {. }
$$


Then we deduce from Hölder's inequality that

$$
\sum_{i=j+1}^{j+N} K_{i} \leq N^{1 / q} \frac{A \omega(1)}{N^{\alpha / p}} \leq A \omega(1) .
$$

We now use the assumption that $X$ is quasi-reflexive, so that $X^{* *}=X \oplus F$, where $F$ is of finite dimension. Thus, for each $\left(n_{1}, \ldots, n_{i}\right) \in[\mathbb{N}] \leq k$, we can decompose $z\left(n_{1}, \ldots, n_{i}\right)=x\left(n_{1}, \ldots, n_{i}\right)+e\left(n_{1}, \ldots, n_{i}\right)$, with $x\left(n_{1}, \ldots, n_{i}\right) \in$ $X$ and $e\left(n_{1}, \ldots, n_{i}\right) \in F$. Then, the compactness of bounded sets in $F$ and another application of Proposition 2.7 allows us to assume, after passing to a full subtree, that

$$
\forall i \in\{1, \ldots, k\} \forall \bar{n}, \bar{v} \in[\mathbb{N}]^{i}, \quad\|e(\bar{n})-e(\bar{v})\|<\eta,
$$

Which implies that for all $i \in\{1, \ldots, k\}$ and all $\bar{n}, \bar{v} \in[\mathbb{N}]^{i}$ we have

$$
|\|z(\bar{n})-z(\bar{v})\|-\|x(\bar{n})-x(\bar{v})\||<\eta \text {. }
$$

We are now ready for the last step of the proof, where we shall build $\bar{m}$ and $\bar{u}$ in $[\mathbb{N}]^{k}$ so that $d_{\mathbb{K}}^{k}(\bar{m}, \bar{u})=N$, but $|f(\bar{m})-f(\bar{u})|$ is bounded by a constant depending only on $\omega(1)$ and on $X$. This will yield a contradiction with the fact $\lim _{N \rightarrow \infty} \rho(N)=\infty$.

First, we set $m_{i}=u_{i}=i$, for all $1 \leq i \leq j$. Then, for $j+1 \leq i \leq j+N$, we set $m_{i}=i$ and $u_{i}=i+N$. Finally, we shall build $m_{i}=u_{i}$ inductively, for $j+N<i \leq k$. Note, that when this will be done, we will indeed have $d_{\mathbb{K}}^{k}(\bar{m}, \bar{u})=N$.

First, we obviously have

$$
\sum_{i=1}^{j} z\left(m_{1}, \ldots, m_{i}\right)-z\left(u_{1}, \ldots, u_{i}\right)=0 .
$$

The next estimate follows from (4.5).

$$
\left|\sum_{i=j+1}^{j+N} z\left(m_{1}, \ldots, m_{i}\right)-z\left(u_{1}, \ldots, u_{i}\right)\right| \leq \sum_{i=j+1}^{j+N} 2\left(K_{i}+\eta\right) \leq 3 A \omega(1),
$$

if $\eta$ was initially chosen small enough.

We now select the remaining coordinates of $\bar{m}$ and $\bar{u}$ inductively using the fact that \|\| is $p$-AUS. To shorten the notation for the end of the proof, we shall now denote $x_{i}=x\left(m_{1}, \ldots, m_{i}\right), z_{i}=z\left(m_{1}, \ldots, m_{i}\right), x_{i}^{\prime}=x\left(u_{1}, \ldots, u_{i}\right)$ and $z_{i}^{\prime}=z\left(u_{1}, \ldots, u_{i}\right)$. First, we simply set $m_{j+N+1}=u_{j+N+1}=j+$ $2 N+1$. We now use the fact that the tree $(z(\bar{m}))_{\bar{m} \in[\mathbb{N}] \leq k}$ is weak ${ }^{*}$-null and Lemma 3.7 (ii) to find $m_{j+N+2}=u_{j+N+2}>j+2 N+1$ such that

$$
\begin{aligned}
& \left\|x_{j+N+1}-x_{j+N+1}^{\prime}+z_{j+N+2}-z_{j+N+2}^{\prime}\right\| \\
& \leq N_{2}^{\bar{\rho}_{X}}\left(\left\|x_{j+N+1}-x_{j+N+1}^{\prime}\right\|,\left\|z_{j+N+2}-z_{j+N+2}^{\prime}\right\|\right)+\eta
\end{aligned}
$$

It follows from (4.6) that

$$
\begin{aligned}
\left\|z_{j+N+1}-z_{j+N+1}^{\prime}+z_{j+N+2}-z_{j+N+2}^{\prime}\right\| \\
\quad \leq N_{2}^{\bar{\rho}_{X}}\left(\left\|z_{j+N+1}-z_{j+N+1}^{\prime}\right\|+\eta,\left\|z_{j+N+2}-z_{j+N+2}^{\prime}\right\|\right)+2 \eta \\
\quad \leq N_{2}^{\bar{\rho}_{X}}\left(\frac{2}{b}\left(K_{j+N+1}+\eta\right)+\eta, \frac{2}{b}\left(K_{j+N+2}+\eta\right)\right)+2 \eta .
\end{aligned}
$$


Similarly, we can inductively find $m_{j+N+2}=u_{j+N+2}<\cdots<m_{k}=u_{k}$ such that,

$$
\left\|\sum_{i=j+N+1}^{k}\left(z_{i}-z_{i}^{\prime}\right)\right\| \leq \frac{2}{b} N_{k-j-N}^{\bar{\rho}_{X}}\left(K_{j+N+1}, \ldots, K_{k}\right)+\omega(1)
$$

provided $\eta$ is chosen small enough. Since Lemma 3.6 ensures the existence of $C>0$ such that $N_{n}^{\bar{\rho}_{X}} \leq C\|\|_{\ell_{n}^{n}}$ for all $n \in \mathbb{N}$ the above inequality yields

$$
\left\|\sum_{i=j+N+1}^{k}\left(z_{i}-z_{i}^{\prime}\right)\right\| \leq \frac{2 C}{b}\left(\sum_{i=j+N+1}^{k} K_{i}^{p}\right)^{1 / p}+\omega(1) \leq\left(\frac{2 C A}{b}+1\right) \omega(1) .
$$

Finally, combining the above estimate with (4.7) and (4.8), we get that

$$
\|f(\bar{m})-f(\bar{u})\| \leq \frac{3 A+2 C A+b}{b} \omega(1) .
$$

As announced at the beginning of the proof, this yields a contradiction if $N$ was initially chosen, as it was possible, so that $\rho(N)>\frac{3 A+2 C A+b}{b} \omega(1)$.

Unlike reflexivity, quasi-reflexivity itself is not enough to prevent the Kalton graphs from embedding into a Banach space. We thank P. Motakis for showing us the next example.

Proposition 4.3 (Motakis). There exists a quasi-reflexive Banach space $X$ such that the family of graphs $\left([\mathbb{N}]^{k}, d_{\mathbb{K}}^{k}\right)_{k \in \mathbb{N}}$ equi-Lipschitz embeds into $X$.

Proof. The proof relies on the existence of a quasi-reflexive Banach space $X$ of order one which admits a spreading model, generated by a basis of $X$ that is equivalent to the summing basis $\left(s_{n}\right)_{n=1}^{\infty}$ of $c_{0}$. This is shown in [9] (Proposition 3.2) and based on a construction given in [6]. We refer the reader to [5] for the necessary definitions. Consequently, there exists a sequence $\left(x_{n}\right)_{n=1}^{\infty}$ in $S_{X}$ and constants $A, B>0$ such that for all $k \leq n_{1}<$ $\cdots<n_{k}$ and all $\varepsilon_{1}, \ldots, \varepsilon_{k}$ in $\{-1,0,1\}$ one has

$$
A\left\|\sum_{i=1}^{k} \varepsilon_{i} s_{i}\right\|_{c_{0}} \leq\left\|\sum_{i=1}^{k} \varepsilon_{i} x_{n_{i}}\right\|_{X} \leq B\left\|\sum_{i=1}^{k} \varepsilon_{i} s_{i}\right\|_{c_{0}} .
$$

For $k \in \mathbb{N}$ and $\bar{n}=\left(n_{1}, \ldots, n_{k}\right) \in[\mathbb{N}]^{k}$ we define

$$
g_{k}(\bar{n})=\sum_{i=1}^{k} x_{2 k+n_{i}} .
$$

It follows easily from Proposition 2.5, the inequality (4.9) and the fact that $\left(s_{n}\right)_{n=1}^{\infty}$ is a spreading sequence that

$$
\frac{A}{2} d_{\mathbb{K}}^{k}(\bar{n}, \bar{m}) \leq\left\|g_{k}(\bar{n})-g_{k}(\bar{m})\right\|_{X} \leq B d_{\mathbb{K}}^{k}(\bar{n}, \bar{m})
$$

for all $\bar{n}, \bar{m} \in[\mathbb{N}]^{k}$.

Remark 4.4. Let us mention that, more generally, it is proved in 2] that for any conditional normalized spreading sequence $\left(e_{n}\right)_{n=1}^{\infty}$, there exists a quasi-reflexive Banach space $X$ of order 1 with a normalized basis $\left(x_{i}\right)_{i=1}^{\infty}$ which generates $\left(e_{n}\right)_{n=1}^{\infty}$ as a spreading model. 


\section{The James Sequence SPACES}

Let $p \in(1, \infty)$. We now recall the definition and some basic properties of the James space $\mathcal{J}_{p}$. We refer the reader to [1](Section 3.4) and references therein for more details on the classical case $p=2$. The James space $\mathcal{J}_{p}$ is the real Banach space of all sequences $x=(x(n))_{n \in \mathbb{N}}$ of real numbers with finite $p$-variation and verifying $\lim _{n \rightarrow \infty} x(n)=0$. The space $\mathcal{J}_{p}$ is endowed with the following norm

$$
\|x\|_{\mathcal{J}_{p}}=\sup \left\{\left(\sum_{i=1}^{k-1}\left|x\left(p_{i+1}\right)-x\left(p_{i}\right)\right|^{p}\right)^{1 / p}: 1 \leq p_{1}<p_{2}<\ldots<p_{k}\right\} .
$$

This is the historical example, constructed for $p=2$ by R.C. James in [11, of a quasi-reflexive Banach space which is isomorphic to its bidual. In fact $\mathcal{J}_{p}^{* *}$ can be seen as the space of all sequences $x=(x(n))_{n \in \mathbb{N}}$ of real numbers with finite $p$-variation, which is $\mathcal{J}_{p} \oplus \mathbb{R} \mathbb{1}$, where $\mathbb{1}$ denotes the constant sequence equal to 1.

The standard unit vector basis $\left(e_{n}\right)_{n=1}^{\infty}\left(e_{n}(i)=1\right.$ if $i=n$ and $e_{n}(i)=0$ otherwise) is a monotone shrinking basis for $\mathcal{J}_{p}$. Hence, the sequence $\left(e_{n}^{*}\right)_{n=1}^{\infty}$ of the associated coordinate functionals is a basis of its dual $\mathcal{J}_{p}^{*}$. Then the weak* topology $\sigma\left(\mathcal{J}_{p}^{*}, \mathcal{J}_{p}\right)$ is easy to describe. A sequence $\left(x_{n}^{*}\right)_{n=1}^{\infty}$ in $\mathcal{J}_{p}^{*}$ converges to 0 in the $\sigma\left(\mathcal{J}_{p}^{*}, \mathcal{J}_{p}\right)$ topology if and only if it is bounded and $\lim _{n \rightarrow \infty} x_{n}^{*}(i)=0$ for every $i \in \mathbb{N}$.

For $x \in \mathcal{J}_{p}$, we define $\operatorname{supp} x=\{i \in \mathbb{N}: x(i) \neq 0\}$. For $x, y \in \mathcal{J}_{p}$, we denote: $x \prec y$ whenever $\max \operatorname{supp} x<\min \operatorname{supp} y$.

Similarly, an element $x^{*}$ of $\mathcal{J}_{p}^{*}$ will be written $x^{*}=\sum_{n=1}^{\infty} x^{*}(n) e_{n}^{*}$ and $\operatorname{supp} x^{*}=\left\{i \in \mathbb{N}: x^{*}(i) \neq 0\right\}$ and we shall denote $x^{*} \prec y^{*}$ whenever $\max \operatorname{supp} x^{*}<\min \operatorname{supp} y^{*}$.

The detailed proof of the following proposition can be found in [20] (Proposition 2.3). This a consequence of the following fact: there exists $C \geq 1$ such that $\left\|\sum_{i=1}^{n} x_{i}\right\|_{\mathcal{J}_{p}}^{p} \leq C \sum_{i=1}^{n}\left\|x_{i}\right\|_{\mathcal{J}_{p}}^{p}$, for all $x_{1} \prec \ldots \prec x_{n}$ in $\mathcal{J}_{p}$.

Proposition 5.1. There exists an equivalent norm || on $\mathcal{J}_{p}$ such that its dual norm ||$_{*}$ has the following property. For any $x^{*}, y^{*} \in J_{p}^{*}$ such that $x^{*} \prec y^{*}$, we have that

$$
\left|x^{*}+y^{*}\right|_{*}^{q} \geq\left|x^{*}\right|_{*}^{q}+\left|y^{*}\right|_{*}^{q} .
$$

In particular, ||$_{*}$ is $q$-AUC $C^{*}$ for the weak $k^{*}$ topology induced by $\mathcal{J}_{p}$ and therefore $\mid$ is $p$-AUS on $\mathcal{J}_{p}$.

There is also a natural weak* topology on $\mathcal{J}_{p}$. Indeed, the summing basis $\left(s_{n}\right)_{n=1}^{\infty}\left(s_{n}(i)=1\right.$ if $i \leq n$ and $s_{n}(i)=0$ otherwise) is a monotone and boundedly complete basis for $\mathcal{J}_{p}$. Thus, $\mathcal{J}_{p}$ is naturally isometric to a dual Banach space: $\mathcal{J}_{p}=X^{*}$ with $X$ being the closed linear span of the biorthogonal functionals $\left(e_{n}^{*}-e_{n+1}^{*}\right)_{n=1}^{\infty}$ in $\mathcal{J}_{p}^{*}$ associated with $\left(s_{n}\right)_{n=1}^{\infty}$. Note that $X=\left\{x^{*} \in \mathcal{J}_{p}^{*}, \sum_{n=1}^{\infty} x^{*}(n)=0\right\}$. Thus, a sequence $\left(x_{n}\right)_{n=1}^{\infty}$ in $\mathcal{J}_{p}$ converges to 0 in the $\sigma\left(\mathcal{J}_{p}, X\right)$ topology if and only if it is bounded and $\lim _{n \rightarrow \infty}\left(x_{n}(i)-x_{n}(j)\right)=0$ for every $i \neq j \in \mathbb{N}$. The next proposition is easy (see Proposition 2.3 in [17] for the case $p=2$ ). 
Proposition 5.2. The usual norm on $\mathcal{J}_{p}$ is $p-A U C^{*}$ for the weak $k^{*}$ topology induced by $X$. In other words, the restriction to $X$ of the usual norm on $\mathcal{J}_{p}^{*}$ is $q$-AUS.

Then, since $X$ is one codimensional in $\mathcal{J}_{p}^{*}$, we have that $\mathcal{J}_{p}^{*}$ is isomorphic to $X \oplus \mathbb{R}$ and therefore also admits an equivalent $q$-AUS norm.

The above remarks combined with Corollary 4.2 immediately yield the following.

Corollary 5.3. Let $p \in(1, \infty)$. Then, the family $\left([\mathbb{N}]^{k}, d_{\mathbb{K}}^{k}\right)_{k \in \mathbb{N}}$ does not equi-coarsely embed into $\mathcal{J}_{p}$, nor does it equi-coarsely embed into $\mathcal{J}_{p}^{*}$.

\section{A Remark on the James tree spaCe}

Let us recall the construction of the James tree space $\mathcal{J T}$. We denote $T=2^{<\omega}$ the tree of all finite sequences with coefficients in $\{0,1\}$ equipped with its natural order: for $s, t \in T$, we say that $s \leq t$ if the sequence $t$ extends $s$. The set of all infinite sequences with coefficients in $\{0,1\}$ will be denoted $2^{\omega}$. For $s \in T$, the length of $s$ is denoted $|s|$. We call segment of $T$ any set of the form $\left\{s \in T, t \leq s \leq t^{\prime}\right\}$ with $t \leq t^{\prime}$ in $T$. For a map $x: T \rightarrow \mathbb{R}$, we define

$$
\|x\|_{\mathcal{J} \mathcal{T}}=\sup \left\{\left(\sum_{i=1}^{n}\left(\sum_{s \in S_{i}} x(s)\right)^{2}\right)^{1 / 2}\right\},
$$

where the supremum is taken over all pairwise disjoint segments $S_{1}, \ldots, S_{n}$ of $T$. Then the James tree space is the space $\mathcal{J} \mathcal{T}=\left\{x: T \rightarrow \mathbb{R},\|x\|_{\mathcal{J T}}<\infty\right\}$ equipped with the norm \|\|$_{\mathcal{J T}}$. For $s \in T$, we denote $e_{s}: T \rightarrow \mathbb{R}$ defined by $e_{s}(t)=\delta_{s, t}, t \in T$. If $\psi: \mathbb{N} \rightarrow T$ is a bijection such that $|\psi(n)| \leq|\psi(m)|$ whenever $n \leq m$, then $\left(e_{\psi(n)}\right)_{n=1}^{\infty}$ is a normalized, monotone and boundedly complete basis of $\mathcal{J T}$. For $s \in T$, the coordinate functional $e_{s}^{*}$ is defined by $e_{s}^{*}(x)=x(s), x \in \mathcal{J} \mathcal{T}$. Then the closed linear span of $\left\{e_{s}^{*}, s \in T\right\}$ in $\mathcal{J T}^{*}$ is denoted $\mathcal{B}$ and $\mathcal{B}^{*}$ is isometric to $\mathcal{J} \mathcal{T}$. The space $\mathcal{J} \mathcal{T}$ was built by R.C. James in [12] to serve as the first example of a separable Banach space with non separable dual, which does not contain an isomorphic copy of $\ell_{1}$.

In [14] it is shown that if a Banach space $X$ coarsely contains $c_{0}$ then there exists $k \in \mathbb{N}$ such that $X^{(k)}$, the dual of order $k$ of $X$, is non separable. A close look at the proof of Theorem 3.5 in [14] allows to state the following.

Theorem 6.1 (Kalton). Let $X$ and $Y$ be two Banach spaces such that $X$ coarsely embeds into $Y$. Assume moreover that there exist an uncountable set $I$ and for every $i \in I$ and $k \in \mathbb{N}$, a 1-Lipschitz map $\left.f_{i}^{k}:\left([\mathbb{N}]^{k}, d_{\mathbb{K}}^{k}\right)\right) \rightarrow X$ such that

$$
\lim _{k \rightarrow \infty} \inf _{i \neq j \in I} \inf _{\mathbb{M} \in[\mathbb{N}]^{\omega}} \sup _{\bar{n} \in[\mathbb{M}]^{k}}\left\|f_{i}^{k}(\bar{n})-f_{j}^{k}(\bar{n})\right\|=\infty .
$$

Then there exists $r \in \mathbb{N}$ such that $Y^{(r)}$ is not separable.

As an application, we can show the following.

Theorem 6.2. Let $Y$ be a Banach space such that $\mathcal{B}$ or $\mathcal{J} \mathcal{T}$ coarsely embeds into $Y$. Then there exists $r \in \mathbb{N}$ such that $Y^{(r)}$ is not separable. 
Proof. For $\sigma \in 2^{\omega}$, we denote $\sigma_{\mid n}=\left(\sigma_{1}, \ldots, \sigma_{n}\right)$. Then, for $k \in \mathbb{N}$, we define $f_{\sigma}^{k}:[\mathbb{N}]^{k} \rightarrow \mathcal{B}$ as follows. For $\bar{n}=\left(n_{1}, \ldots, n_{k}\right) \in[\mathbb{N}]^{k}$ let

$$
f_{\sigma}^{k}(\bar{n})=\frac{1}{\sqrt{k}} \sum_{i=1}^{k} \sum_{s \leq \sigma_{\mid n_{i}}} e_{s}^{*} .
$$

Assume for instance that $n_{1} \leq m_{1} \leq \cdots n_{k} \leq m_{k}$. Then we can write

$$
f_{\sigma}^{k}(\bar{m})-f_{\sigma}^{k}(\bar{n})=\frac{1}{\sqrt{k}} \sum_{i=1}^{k} \sum_{s \in S_{i}} e_{s}^{*},
$$

where $S_{1}, \ldots, S_{k}$ are pairwise disjoint segments in $T$. Note that for any segment $S_{i}$ the sum $\sum_{s \in S_{i}} e_{s}^{*}$ belongs to the unit ball of $\mathcal{J T}^{*}$. It then follows from the Cauchy-Schwarz inequality that $f_{\sigma}^{k}$ is 1 -Lipschitz on $\left([\mathbb{N}]^{k}, d_{\mathbb{K}}^{k}\right)$. Assume now that $\sigma \neq \tau \in 2^{\omega}$. Pick $r \in \mathbb{N}$ such that $\sigma_{r} \neq \tau_{r}$. Then for any $\mathbb{M} \in[\mathbb{N}]^{\omega}$ and any $\bar{n}=\left(n_{1}, \ldots, n_{k}\right) \in[\mathbb{M}]^{k}$ with $n_{1} \geq r$, we have

$$
\left\|f_{\sigma}^{k}(\bar{n})-f_{\tau}^{k}(\bar{n})\right\|_{\mathcal{B}} \geq\left|\left\langle f_{\sigma}^{k}(\bar{n})-f_{\tau}^{k}(\bar{n}), e_{\sigma_{\mid n_{1}}}\right\rangle\right| \geq \sqrt{k} .
$$

By Theorem 6.1 and the uncountability of $2^{\omega}$, this finishes our proof for $\mathcal{B}$.

For $\sigma \in 2^{\omega}$ and $k \in \mathbb{N}$ define now $g_{\sigma}^{k}:[\mathbb{N}]^{k} \rightarrow \mathcal{J} \mathcal{T}$ by

$$
\forall \bar{n}=\left(n_{1}, \ldots, n_{k}\right) \in[\mathbb{N}]^{k}, g_{\sigma}^{k}(\bar{n})=\frac{1}{\sqrt{2 k}} \sum_{i=1}^{k} e_{\sigma_{\mid n_{i}}} .
$$

It is easily checked that $g_{\sigma}^{k}$ is 1 -Lipschitz on $\left([\mathbb{N}]^{k}, d_{\mathbb{K}}^{k}\right)$. Assume now that $\sigma \neq \tau \in 2^{\omega}$. Pick $r \in \mathbb{N}$ such that $\sigma_{r} \neq \tau_{r}$. Then for any $\mathbb{M} \in[\mathbb{N}]^{\omega}$ and any $\bar{n}=\left(n_{1}, \ldots, n_{k}\right) \in[\mathbb{M}]^{k}$ with $n_{1} \geq r$, denote $S=\left\{s \in T, \sigma_{\mid n_{1}} \leq s \leq \sigma_{\mid n_{k}}\right\}$. The set $S$ is a segment in $T$ and $x^{*}=\sum_{s \in S} e_{s}^{*}$ is in the unit ball of $\mathcal{J}^{*}$. Therefore

$$
\left\|g_{\sigma}^{k}(\bar{n})-g_{\tau}^{k}(\bar{n})\right\|_{\mathcal{J} \mathcal{T}} \geq\left\langle g_{\sigma}^{k}(\bar{n})-g_{\tau}^{k}(\bar{n}), x^{*}\right\rangle \geq \frac{\sqrt{k}}{\sqrt{2}}
$$

This concludes our proof for $\mathcal{J T}$.

Acknowledgements. We are grateful to P. Motakis for allowing us to include Proposition 4.3 and for very useful discussions. We also wish to mention that the formula describing the interlaced distance given in Proposition 2.2 has been independently obtained by F. Baudier, P. Motakis and Th. Schlumprecht.

\section{REFERENCES}

[1] F. Albiac and N.J. Kalton, Topics in Banach Space Theory, Second Edition, volume 233 of Graduate Texts in Mathematics, Springer, New York, (2006-2016).

[2] S. Argyros, P. Motakis and B. Sari, A study of conditional spreading sequences, preprint arXiv: $1611.04443 \mathrm{v} 1$.

[3] F. Baudier, G. Lancien, P. Motakis and Th. Schlumprecht, A new coarsely rigid class of Banach spaces, preprint arXiv:1806.00702.

[4] F. Baudier, G. Lancien and Th. Schlumprecht, The coarse geometry of Tsirelson's space and applications, J. Amer. Math. Soc., 31 (2018), no. 3, 699-717.

[5] B. Beauzamy and J.T. Lapresté, Modèles étalés des espaces de Banach, Travaux en Cours, Hermann, Paris, (1984). 
[6] S.F. Bellenot, R. Haydon, and E. Odell, Quasi-reflexive and tree spaces constructed in the spirit of R.C. James, Contemp. Math., Amer. Math. Soc., Providence, RI, 85 (1989), 19-43.

[7] R.M. Causey and G. Lancien, Prescribed Szlenk index of separable Banach spaces, to appear in Studia Math., preprint arXiv: 1710.01638.

[8] S. Dilworth, D. Kutzarova, G. Lancien and L. Randrianarivony, Equivalent norms with the property $(\beta)$ of Rolewicz, Rev. R. Acad. Cienc. Exactas, Fís. Nat. Ser. A Mat. RACSAM, 111 (2017), no. 1, 101-113.

[9] D. Freeman, E. Odell, B. Sari, and B. Zheng, On spreading sequences and asymptotic structures, to appear in Trans. Amer. Math. Soc., preprint arXiv: 1607.03587v1.

[10] W.T. Gowers, Ramsey Methods in Banach Spaces, in Handbook of the Geometry of Banach Spaces vol. 2, 2003, 1071-1097.

[11] R.C. James, Bases and reflexivity of Banach spaces, Ann. of Math. (2), 52, (1950). $518-527$.

[12] R.C. James, A separable somewhat reflexive Banach space with nonseparable dual, Bull. Amer. Math. Soc., 80, (1974), 738-743.

[13] W.B. Johnson, J. Lindenstrauss, D. Preiss and G. Schechtman, Almost Fréchet differentiability of Lipschitz mappings between infinite-dimensional Banach spaces, Proc. London Math. Soc. (3) 84 (2002), no. 3, 711-746.

[14] N.J. Kalton, Coarse and uniform embeddings into reflexive spaces, Quart. J. Math. (Oxford), 58 (2007), 393-414.

[15] N.J. Kalton and L. Randrianarivony, The coarse Lipschitz geometry of $\ell_{p} \oplus \ell_{q}$, Math. Ann. 341 (2008), no. 1, 223-237.

[16] N.J Kalton, Uniform homeomorphisms of Banach spaces and asymptotic structure, Trans. Amer. Math. Soc., 365 (2013), 1051-1079.

[17] G. Lancien, Réflexivité et normes duales possèdant la propriété uniforme de KadecKlee, Publications Mathématiques de Besançon, 14, 1993/94.

[18] G. Lancien and M. Raja, Asymptotic and coarse Lipschitz structures of quasi-reflexive Banach spaces, Houston J. of Math, 44 (2018) no 3, 927-940.

[19] V.D. Milman, Geometric theory of Banach spaces. II. Geometry of the unit ball (Russian), Uspehi Mat. Nauk 26 (1971), 73-149. English translation: Russian Math. Surveys 26 (1971), 79-163.

[20] F. Nétillard, Coarse Lipschitz embeddings of James spaces, Bull. Belg. Math. Soc. Simon Stevin, 25 (2018), 1-14.

[21] I. J. Schoenberg, Metric spaces and positive definite functions, Trans. Am. Math. Soc. 44 (1938), 522-536.

Gilles Lancien, Laboratoire de Mathématiques de Besançon, Université Bourgogne Franche-Comté, CNRS UMR-6623, 16 route de Gray, 25030 Besançon Cédex, Besançon, France

E-mail address: gilles.lancien@univ-fcomte.fr

Colin Petitjean, Laboratoire de Mathématiques de Besançon, Université Bourgogne Franche-Comté, CNRS UMR-6623, 16 route de Gray, 25030 Besançon Cédex, Besançon, France

E-mail address: colin.petitjean@univ-fcomte.fr

Antonin Procházka, Laboratoire de Mathématiques de Besançon, Université Bourgogne Franche-Comté, CNRS UMR-6623, 16 route de Gray, 25030 Besançon Cédex, Besançon, France

E-mail address: antonin.prochazka@univ-fcomte.fr 